\title{
Metabolic Control of Th17 Cell Generation and CNS Inflammation
}

\section{Kai Yang and Hongbo $\mathrm{Chi}^{*}$}

Department of Immunology, St. Jude Children's Research Hospital, Memphis, TN 38105, USA

\begin{abstract}
Multiple sclerosis (MS), an inflammatory demyelinating disease of the central nervous system (CNS), results from uncontrolled auto reactive T cells that infiltrate the CNS and attack the myelin sheath. Th17 cells play a prominent role in the pathogenesis of MS and experimental autoimmune encephalomyelitis (EAE), a mouse model of MS. Extensive studies have focused on understanding the roles of cytokine signaling and transcriptional network in the differentiation of Th17 cells and their pathogenicity in CNS inflammation. Aside from these events, activated T cells dynamically reprogram their metabolic pathways to fulfill the bioenergic and biosynthetic requirements for proper $\mathrm{T}$ cell functions. Emerging evidence indicates that modulation of these metabolic pathways impinges upon the differentiation of Th17 cells and the pathogenesis of EAE. Thus, a better understanding of the functions and mechanisms of $\mathrm{T}$ cell metabolism in Th17 cell biology may provide new avenues for therapeutic targeting of MS. In this review, we discuss the recent advances in our understanding of T cell metabolic pathways involved in Th17 cell differentiation and CNS inflammation.
\end{abstract}

Keywords: CNS inflammation; Autoimmune disease; $\mathrm{T}$ cell metabolism

\section{Introduction}

To ensure proper electrical activity of neurons, the central nervous system (CNS) strictly controls immune cell entry across the bloodbrain barrier (BBB). Multiple sclerosis (MS) is an autoimmune disease initiated by auto reactive T cells, which break down BBB and gain the access to CNS where they cause inflammation and demyelination. MS is one of the most common idiopathic inflammatory demyelinating diseases in young adults, affecting more than 2.5 million people worldwide. On the basis of clinical symptoms and course, MS is divided into relapsing remitting (RR), primary progressive (PP), and secondary progressive (SP) subtypes [1].

The principal animal model of MS, experimental sclerosis encephalomyelitis (EAE), has been widely utilized to investigate the function of myelin antigen-specific CD4+ T helper (Th) cells that mediate dysregulated immune functions in the CNS. Based on unique cytokine production, effector Th cells are categorized into various subsets, such as Th1, Th2 and Th17 cells, which are implicated in distinct protective and pathogenic immune responses [2]. Although Th1 cells were originally considered as the pathogenic effector cells for neuroinflammation, recent studies indicate that Th17 cells play a key role in the pathogenesis of MS [3]. Th17 cells are characterized by producing IL-17A, IL-17F, IL-21 and IL-22, and mediate protective functions for the clearance of extracellular pathogens during infection [3]. The differentiation of Th17 cells can be induced by proinflammatory cytokines in TGF- $\beta$ dependent and independent ways $[4,5]$. The proinflammatory cytokine IL-6, together with TGF- $\beta$, drive the expression of Th17-specific transcription factor ROR $\gamma t$ and the differentiation of Th17 cells. In the absence of TGF- $\beta$, a combination of proinflammatory cytokines IL-1 $\beta$, IL-6 and IL-23 induces differentiation of Th17 cells and promote the pathogenesis of EAE [5]. Blockade of TGF- $\beta$ or IL-23 signaling markedly suppresses Th17 differentiation and ameliorates EAE progression [6,7]. Moreover, genome-wide associated studies of MS patients reveal that several genetic risk factors are associated with IL-23/Th17 axis [1]. Recent studies also uncovered the critical role of the cytokine granulocytemacrophage colony-stimulating factor (GM-CSF) in IL-23-mediated
EAE pathogenesis. IL-23 induces the expression of GM-CSF by Th17 cells, which in turn enhances the production of IL-23 by dendritic cells (DCs) [8,9]. This IL-23-Th17-GM-CSF circuit promotes both sustained Th17 responses and the de novo generation of pathogenic Th17 cells. These studies indicate that the complex interaction of various cytokines and inflammatory factors dictates the pathogenicity of Th17 cells and disease outcome.

Aside from the regulation by cytokine signaling, emerging evidence highlights that $\mathrm{T}$ cell metabolism is an important regulator of $\mathrm{T}$ cell fate decisions and functions. To meet the bioenergetic and biosynthetic demands at various states, including development, maintenance, activation and differentiation, $\mathrm{T}$ cells dynamically reprogram cellular metabolic pathways [10-13]. In the quiescent state, naïve T cells generate ATP through catabolic metabolism, including oxidative phosphorylation (OXPHOS) of glucose, amino acid and lipids in mitochondria. In contrast, antigen-primed $\mathrm{T}$ cells switch from catabolism to anabolism, characterized by marked upregulation of glycolysis, glutaminolysis and biosynthetic activities, for rapid clonal expansion [14]. Distinct metabolic requirements have also been implicated in the control of different $\mathrm{T}$ cell subsets for their specific functions in immunity and inflammation $[15,16]$. Conversely, modulation of specific metabolic pathways can suppress pathogenic $\mathrm{T}$ cell responses, for example, the differentiation of Th17 cells and EAE pathogenesis [16]. In this review, we discuss the current understanding of the regulation, function and targeting of $\mathrm{T}$ cell metabolism and the associated regulatory pathways in Th17 cell differentiation and CNS inflammation (Figure 1).

*Corresponding author: Hongbo Chi, Department of Immunology, St. Jude Children's Research Hospital, Memphis, TN 38105, USA, Tel: 901-595-6282; Fax: 901-595-5766; E-mail: hongbo.chi@stjude.org

Received January 08, 2014; Accepted January 24, 2014; Published February 05,2014

Citation: Yang K, Chi H (2014) Metabolic Control of Th17 Cell Generation and CNS Inflammation. J Neurol Neurophysiol S12: 004. doi:10.4172/2155-9562.S12004

Copyright: ( 2014 Yang K, et al. This is an open-access article distributed under the terms of the Creative Commons Attribution License, which permits unrestricted use, distribution, and reproduction in any medium, provided the original author and source are credited. 


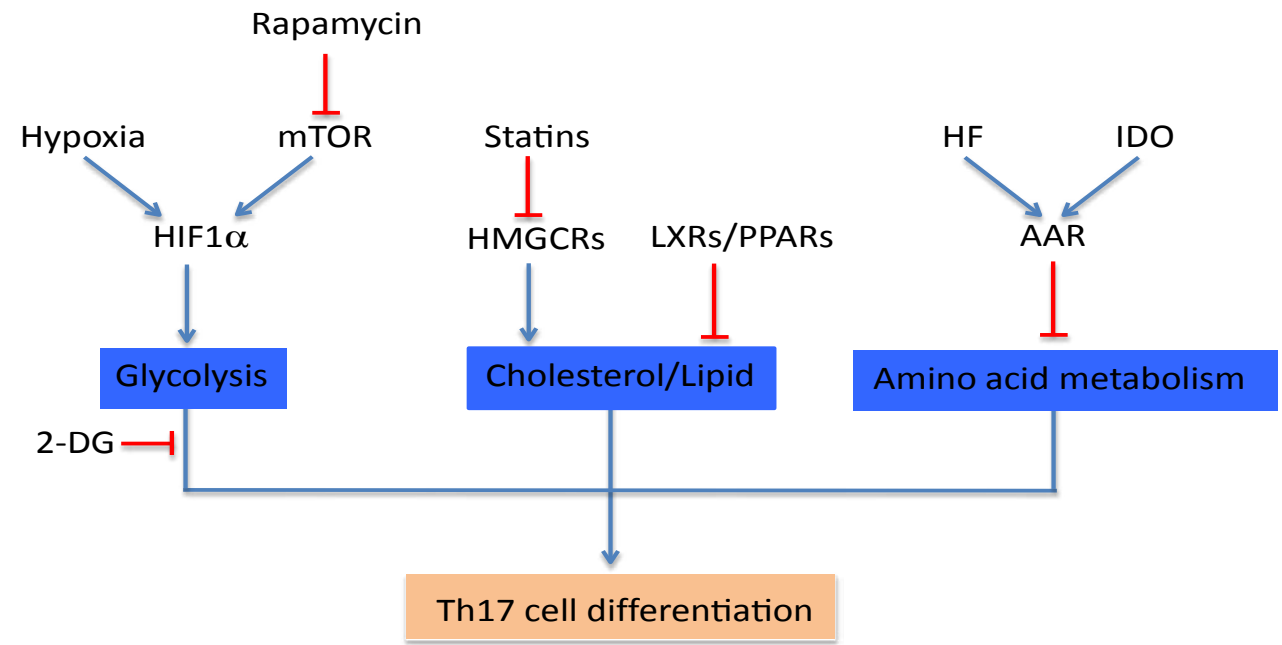

Figure 1: Regulation of Th17 cell differentiation by nutrient metabolism, including glucose, cholesterol and amino acid metabolism.

\section{Glucose Metabolism and the mTOR-HIF1a Signaling Axis}

Glucose metabolism provides critical bioenergetic and biosynthetic support for proper $\mathrm{T}$ cell functions. Quiescent $\mathrm{T}$ cells catabolically utilize glucose-derived pyruvate along with lipids and amino acids to generate ATP for their survival. Upon antigen stimulation, activated T cells upregulate glucose transporter Glut l and hence elevate glucose availability. Further, they switch to aerobic glycolysis to provide essential metabolic intermediates for biosynthesis of macromolecules. This metabolic shift is required to meet the increased bioenergetic and biosynthetic demands for rapid clonal expansion during immune responses. Blockade of glucose metabolism markedly suppresses $\mathrm{T}$ cell activation and proliferation $[14,17]$.

Aside from its role in $\mathrm{T}$ cell activation and proliferation, glucose metabolism also impinges upon Th17 cell differentiation and the pathogenesis of EAE. As compared with induced Treg (iTreg) cells, Th17 cells have much higher glycolytic activity, associated with elevated expression of metabolic genes involved in the glycolytic pathway $[15,16]$. Glut1-transgenic T cells enhance glucose uptake and glycolytic activity, and manifest increased IL-17 production in aged mice [17]. Compared with other Th subsets, Th17 cells selectively express the transcription factor HIFla that regulates the expression of genes involved in the glycolytic pathway. The specific ablation of HIF1 $\alpha$ in $\mathrm{T}$ cells reciprocally affects Th17 and iTreg cell generation, indicated by reduced expression of the Th17 family cytokines IL-17A, IL-17F, IL-21 and IL-22, and upregulated expression of Treg-specific transcription factor Foxp3 [16]. Additionally, HIF1a-deficient T cells have defective expression of IL-23R, but maintain expression of Th17 transcription factors ROR- $\gamma t$ and ROR- $\alpha$. Analysis of global gene expression profiles reveals that glycolytic or related metabolic pathways are significantly diminished in HIF1a-deficient $\mathrm{T}$ cells [16]. Similar as HIF1a deficiency, inhibition of glycolysis by the pharmacological inhibitor 2-deoxyglucose (2-DG) also results in reciprocal changes of Th17 and iTreg cell generation [16]. In EAE mice, increased glycolysis has been observed in infiltrating lymphocytes in the spinal cord [18]. Blockade of glycolysis by HIFla deletion or 2-DG treatment ameliorates the development of EAE $[16,19]$. These results demonstrate that HIF1adependent glycolysis functions as a metabolic checkpoint for the differentiation of Th17 cells.
HIF1 $\alpha$ expression in T cells is regulated at both transcriptional and post-transcriptional levels. Shi et al. revealed that signaling via mechanistic target of rapamycin (mTOR) acts as an important upstream pathway to induce HIF1a transcription [16]. mTOR, an evolutionally conserved serine and threonine kinase, has been recently implicated in the integration of immune signals and metabolic cues in lymphocyte proliferation, differentiation and survival $[10,11,13]$. Rapamycin, a specific mTOR inhibitor, inhibits the transcription of HIF1a. Similar as HIF1a deficiency, treatment of cells with rapamycin suppresses the expression of glycolytic genes and IL-17 [16]. Genetic evidence also indicates the important role of mTOR in Th17 cell differentiation $[20,21]$. In agreement with these observations, rapamycin treatment ameliorates the clinical course of EAE [22-24]. Given the similar effects of blocking mTOR, HIF1 $\alpha$ and glycolysis on Th17 cell differentiation and the relationship between these pathways, these studies highlight the important roles of the mTOR-HIF1a-glycolysis axis in Th17 cell differentiation. The stability of the HIF1 $\alpha$ protein is tightly regulated by hypoxia. Dang et al. described that hypoxia-stabilized HIF1a promotes Th17 cell differentiation via promoting ROR $\gamma t$ activity and inducing Foxp3 degradation [19]. In agreement with the role of hypoxia in Th17 cell differentiation, a recent study indicates that hypoxia followed by reoxygenation accelerates Th17 cell differentiation in an mTORC1HIFla-dependent manner [25]. These studies indicate that mTOR signaling and hypoxia conditions are critical regulators of HIF1a expression in Th17 cell differentiation, and highlight that modulation of HIFla function or the glycolytic pathway is a potential therapeutic strategy for the treatment of MS patients.

\section{Amino Acid Metabolism and IDO Functions}

$\mathrm{T}$ lymphocytes regulate uptake of essential amino acids from microenvironment to meet the metabolic demands of an immune response $[14,26]$. Blockade of amino acid metabolism in T cells induces metabolic energy, indicated by defective proliferation upon TCR stimulation [27]. The relative abundance of amino acids in the immune microenvironment may therefore establish a fundamental metabolic requirement for proper $\mathrm{T}$ cell-mediated immune responses.

A recent study by Sundrud et al. reported that induction of amino acid starvation response (AAR) by a small molecule compound halofuginone $(\mathrm{HF})$ attenuates Th17 cell differentiation and EAE 
pathogenesis [28]. HF is a derivative of the plant alkaloid febrifugine used in traditional Chinese medicine to reduce malarial fever [29]. Treatment of T cells with HF selectively impairs Th17 cell differentiation without affecting other Th subsets, and reciprocally promotes iTreg generation. HF induces AAR by downregulating expression of genes involved in amino acid transports, biogenesis and protein synthesis. The starvation of amino acids activates the kinase GCN2, which has been recently shown to mediate the remission phase of EAE in mice [30]. Addition of excess amino acids restores the defective Th17 cell differentiation in HF-treated cells. In line with these observations, HF treatment significantly ameliorates the pathogenesis in an active EAE mouse model; however, it cannot provide protection in the passive EAE model, indicating that HF inhibits Th17 cell differentiation, but not the effector function of developed Th17 cells [28].

Metabolism of tryptophan, one of the essential amino acids, by the enzyme indoleamine 2,3-dioxygenase (IDO) plays an important role in the control of EAE pathogenesis. IDO is an inducible tryptophandegrading enzyme, expressed by DCs and other immune cells. IDO causes the breakdown of tryptophan and induces localized amino acid depletion, leading to cytoprotective AAR and activation of GCN2 [31]. IDO promotes immune tolerance in part by affecting generation of T effector cells and iTreg cells [32-34]. Specifically, IDO suppresses the differentiation of Th1 and Th17 cells, but enhances the generation of iTreg cells and maintains Treg cell function $[32,33]$. Consistent with the effects of IDO on T cell differentiation in vitro, IDO expressed by DCs suppresses EAE [34,35]. Moreover, IDO-deficient mice develop more severe EAE, associated with the enhanced generation of Th17 cells but reduced Treg cells [34]. The Tryptophan metabolite 3-hydroxyanthranillic acid (3-HAA) plays an important role in mediating IDO-induced immune tolerance via affecting the generation of Treg cells [34]. Administration of 3-HAA at the early phase of EAE ameliorates the disease progression, but starting the treatment at the peak of EAE has no therapeutic effects [34]. Whereas the underlying mechanism remains to be further investigated, these studies establish the link of regulation of amino acid availability to the fate decisions of Th17 and Treg populations.

\section{Lipid and Cholesterol Metabolism and LXR/PPAR Functions}

Cholesterol is a vital constituent of cell membrane and modulates membrane fluidity and permeability. Activated $\mathrm{T}$ cells markedly enhance cholesterol synthesis, and blockade of cholesterol synthesis suppresses the differentiation of Th17 cells and neuroinflammation. 3-hydroxy-3-methylglutaryl CoA (HMG-CoA) reductase (HMGCR), a rate-limiting enzyme of the mevalonate pathway that produces cholesterol and other isoprenoids, is the molecular target of the cholesterol-lowering drugs statins. Administration of statins inhibits Th17 cell differentiation by directly suppressing the expression of Th17transcription factor ROR $\gamma \mathrm{t}[36,37]$, as well as indirectly by targeting DCs to modulate cytokine expression and antigen presentation $[36,37]$. Mevalonic acid, a downstream metabolite of HMGCR, restores Th17 cell differentiation in statin-treated cells [36,37]. Statins also suppress Th17 cell differentiation in CD4+ T cells derived from patients with multiple sclerosis [38]. In line with these observations, statin treatment ameliorates the pathogenesis of EAE [39-41].

Transcriptional regulators of cholesterol and lipid metabolism have been also implicated in the control of Th17 cell differentiation. Liver X receptors (LXRs), a member of nuclear receptors, are activated by endogenous oxysterols (oxidized derivatives of cholesterol) and thus act as cholesterol sensors. When cellular oxysterols accumulate due to increasing concentration of cholesterol, LXRs are activated and induce expression of genes required to eliminate cellular cholesterol [42]. Systemic administration of LXR agonists GW3965 and T0901317 inhibits Th17 cell differentiation [43,44] and ameliorates EAE progression [44]. In contrast, LXR deficiency promotes the generation of Th17 cells and exacerbates the disease [44]. Another type of nuclear receptors, peroxisome proliferator-activated receptors (PPARs), play a well-defined role in regulating lipid homeostasis, and can function as coactivators of LXRs. PPARs are important negative regulators of Th17 cell differentiation. Accordingly, treatment of mice with synthetic agonists of PPARs ameliorates EAE, and deficiency of PPAR $\gamma$ or PPAR $\delta$ exacerbates EAE pathogenesis [45-47]. These studies highlight that modulation of cholesterol and lipid synthesis is a promising therapeutic approach for MS.

\section{High Salt Diet and SGK1 Functions}

The etiology of MS is driven by multiple factors, such as genetics, immunity and environment. Genetic risk factors are associated with the IL23-Th17 axis that is pivotal for the pathogenicity of Th17 cells and the development of EAE [1]. How environmental factors contribute to these processes remains less understood. Two recent studies reported that high salt diet is an environmental risk factor in the development of EAE through enhancing the pathogenicity of Th17 cells, in a manner dependent upon IL-23R signaling [48,49].

Kleinewietfeld et al. reported that high concentration of $\mathrm{NaCl}$ selectively enhances the differentiation of Th 17 cells, but not Th1 or Th2 cells [48]. Microarray analysis reveals that $\mathrm{NaCl}$ treatment markedly promotes expression of key signature molecules of Th17 cells, including IL-17A, IL-17F, ROR $\gamma$ t, and IL-23R, as well as GM-CSF and CCR6 that mediates the enhanced pathogenicity of Th17 cells. In agreement with the in vitro observations, mice with high-salt diet exhibit accelerated onset and increased severity of EAE, associated with augmented infiltration of Th17 cells into the spinal cord [48]. Mechanistically, increased $\mathrm{NaCl}$ concentration activates the p38 MAPK pathway to enhance the expression of SGK1 and NFAT5, both of which contribute to Th17 cell differentiation, although whether p38 MAPK signaling in $\mathrm{T}$ cells directly regulates Th17 cell generation is unclear [50]. Wu et al also studied the effects of high salt diet on EAE pathogenesis and the underlying molecular basis [49]. From transcriptional profiling of developing Th17 cells, the authors identified SGK1 as a critical signaling node downstream of IL-23R signaling. SGK1 expression is specifically induced and maintained by exposure to IL-23. In turn, SGK1 promotes IL-23R expression through inactivating transcription factor Foxo1, which is an inhibitor of IL-23R expression.

Furthermore, SGK1 deficiency impairs IL-17A expression in response to IL-23 stimulation but not in response to TGF- $\beta$ and IL- 6 stimulation, indicating the critical role of SGK1 in mediating IL23R-dependent stability and maintenance of Th17 cells. Importantly, SGK1 expression is induced by high salt, and loss of SGK1 in T cells protects the mice from high-salt-mediated exacerbation of EAE [49]. These studies establish the pathogenic effects of high salt diet in EAE pathogenesis and the underlying signaling mechanisms. They further highlight that reduced intake of high salt diet may prevent the development of MS, and targeting SGK1 or related molecular pathways may have beneficial effects in MS patients.

\section{Concluding Remarks}

For the development of effective drugs to target autoimmune neuroinflammation, much emphasis is placed on understanding the mechanisms underlying the differentiation and pathogenicity of Th17 
cells. These studies have led to the identification of the pivotal role of the IL-23/Th17/GM-CSF axis in the generation of pathogenic Th17 cells. Emerging evidence also highlights the fundamental importance of $\mathrm{T}$ cell metabolism in the regulation of $\mathrm{T}$ cell responses, such as $\mathrm{T}$ cell survival, proliferation and differentiation. In particular, recent studies have established the roles of specific metabolic pathways in Th17 cell differentiation, and modulation of these pathways manifests therapeutic opportunities to target CNS inflammation. Continued investigation on the interplay of immune signals and metabolic cue and metabolic signatures of pathogenic Th17 cells will likely facilitate the development of novel therapeutic strategies to restore the dysregulated immune responses in MS. At the mechanistic level, the integrated use of mouse genetic and pharmacological approaches and the EAE model will be a powerful strategy to further define the regulation and function of specific metabolic pathways in the generation and pathogenicity of Th17 cells.

\section{Acknowledgements}

This work is supported by US National Institutes of Health (R01 Al101407, R01 NS064599 and R21 Al094089), National Multiple Sclerosis Society, and the American Lebanese Syrian Associated Charities (H.C.), and an Arthritis Foundation Postdoctoral Fellowship (K.Y.).

\section{References}

1. Nylander A, Hafler DA (2012) Multiple sclerosis. J Clin Invest 122: 1180-1188.

2. Nakayamada S, Takahashi H, Kanno Y, O'Shea JJ (2012) Helper T cell diversity and plasticity. Curr Opin Immunol 24: 297-302.

3. Korn T, Bettelli E, Oukka M, Kuchroo VK (2009) IL-17 and Th17 Cells. Annu Rev Immunol 27: 485-517.

4. Ivanov II, McKenzie BS, Zhou L, Tadokoro CE, Lepelley A, et al. (2006) The orphan nuclear receptor RORgammat directs the differentiation program of proinflammatory IL-17+ T helper cells. Cell 126: 1121-1133.

5. Ghoreschi K, Laurence A, Yang XP, Tato CM, McGeachy MJ, et al. (2010) Generation of pathogenic $T(H) 17$ cells in the absence of TGF- $\beta$ signalling. Nature 467: 967-971.

6. Veldhoen M, Hocking RJ, Flavell RA, Stockinger B (2006) Signals mediated by transforming growth factor-beta initiate autoimmune encephalomyelitis, but chronic inflammation is needed to sustain disease. Nat Immunol 7: 1151-1156.

7. McGeachy MJ, Chen Y, Tato CM, Laurence A, Joyce-Shaikh B, et al. (2009) The interleukin 23 receptor is essential for the terminal differentiation of interleukin 17-producing effector T helper cells in vivo. Nat Immunol 10: 314-324.

8. El-Behi M, Ciric B, Dai H, Yan Y, Cullimore M, et al. (2011) The encephalitogenicity of $\mathrm{T}(\mathrm{H}) 17$ cells is dependent on IL-1- and IL-23-induced production of the cytokine GM-CSF. Nat Immunol 12: 568-575.

9. Codarri L, Gyülvészi G, Tosevski V, Hesske L, Fontana A, et al. (2011) RORyt drives production of the cytokine GM-CSF in helper T cells, which is essential for the effector phase of autoimmune neuroinflammation. Nat Immunol 12: 560 567

10. Chi H (2012) Regulation and function of mTOR signalling in T cell fate decisions. Nat Rev Immunol 12: 325-338.

11. Yang $\mathrm{K}$, Chi $\mathrm{H}$ (2012) mTOR and metabolic pathways in T cell quiescence and functional activation. Semin Immunol 24: 421-428.

12. Maclver NJ, Michalek RD, Rathmell JC (2013) Metabolic regulation of $T$ lymphocytes. Annu Rev Immunol 31: 259-283.

13. Zeng H, Chi H (2013) mTOR and lymphocyte metabolism. Curr Opin Immuno 25: 347-355.

14. Wang R, Dillon CP, Shi LZ, Milasta S, Carter R, et al. (2011) The transcription factor Myc controls metabolic reprogramming upon T lymphocyte activation. Immunity 35: 871-882.

15. Michalek RD, Gerriets VA, Jacobs SR, Macintyre AN, Maclver NJ, et al. (2011) Cutting edge: distinct glycolytic and lipid oxidative metabolic programs are essential for effector and regulatory CD4+ T cell subsets. J Immunol 186: 32993303.
6. Shi LZ, Wang R, Huang G, Vogel P, Neale G, et al. (2011) HIF1alpha-dependent glycolytic pathway orchestrates a metabolic checkpoint for the differentiation of TH17 and Treg cells. J Exp Med 208: 1367-1376.

17. Jacobs SR, Herman CE, Maciver NJ, Wofford JA, Wieman HL, et al. (2008) Glucose uptake is limiting in T cell activation and requires CD28-mediated Aktdependent and independent pathways. J Immunol 180: 4476-4486.

18. Radu CG, Shu CJ, Shelly SM, Phelps ME, Witte ON (2007) Positron emission tomography with computed tomography imaging of neuroinflammation in experimental autoimmune encephalomyelitis. Proc Natl Acad Sci U S A 104: 1937-1942.

19. Dang EV, Barbi J, Yang HY, Jinasena D, Yu H, et al. (2011) Control of T(H)17/ $\mathrm{T}(\mathrm{reg})$ balance by hypoxia-inducible factor 1 . Cell 146: 772-784.

20. Delgoffe GM, Kole TP, Zheng Y, Zarek PE, Matthews KL, et al. (2009) The mTOR kinase differentially regulates effector and regulatory $T$ cell lineage commitment. Immunity 30: 832-844.

21. Kurebayashi $Y$, Nagai S, Ikejiri A, Ohtani M, Ichiyama K, et al. (2012) PI3KAkt-mTORC1-S6K1/2 axis controls Th17 differentiation by regulating Gfi1 expression and nuclear translocation of RORY. Cell Rep 1: 360-373.

22. Esposito M, Ruffini F, Bellone M, Gagliani N, Battaglia M, et al. (2010) Rapamycin inhibits relapsing experimental autoimmune encephalomyelitis by both effector and regulatory T cells modulation. J Neuroimmunol 220: 52-63.

23. Lisi L, Navarra P, Cirocchi R, Sharp A, Stigliano E, et al. (2012) Rapamycin reduces clinical signs and neuropathic pain in a chronic model of experimental autoimmune encephalomyelitis. J Neuroimmunol 243: 43-51.

24. Dello Russo C, Lisi L, Feinstein DL, Navarra P (2013) mTOR kinase, a key player in the regulation of glial functions: relevance for the therapy of multiple sclerosis. Glia 61: 301-311.

25. Ikejiri A, Nagai S, Goda N, Kurebayashi Y, Osada-Oka M, et al. (2012) Dynamic regulation of Th17 differentiation by oxygen concentrations. Int Immunol 24 137-146.

26. Sinclair LV, Rolf J, Emslie E, Shi YB, Taylor PM, et al. (2013) Control of Amino-Acid Transport by Antigen Receptors Coordinates the Metabolic Reprogramming Essential for T Cell Differentiation. Nat Immunol 14: 500-508.

27. Zheng Y, Delgoffe GM, Meyer CF, Chan W, Powell JD (2009) Anergic T cells are metabolically anergic. J Immunol 183: 6095-6101.

28. Sundrud MS, Koralov SB, Feuerer M, Calado DP, Kozhaya AE, et al. (2009) Halofuginone inhibits $\mathrm{TH} 17$ cell differentiation by activating the amino acid starvation response. Science 324: 1334-1338.

29. Pines M, Snyder D, Yarkoni S, Nagler A (2003) Halofuginone to treat fibrosis in chronic graft-versus-host disease and scleroderma. Biol Blood Marrow Transplant 9: 417-425

30. Orsini H1, Araujo LP1, Maricato JT1, Guereschi MG1, Mariano M1, et al (2013) GCN2 kinase plays an important role triggering the remission phase of experimental autoimmune encephalomyelitis (EAE) in mice. Brain Behav Immun

31. Mellor AL, Munn DH (2004) IDO expression by dendritic cells: tolerance and tryptophan catabolism. Nat Rev Immunol 4: 762-774.

32. Baban B, Chandler PR, Sharma MD, Pinkala J, Koni PA, et al. (2009) IDO activates regulatory $T$ cells and blocks their conversion into Th17-like T cells. J Immunol 183: 2475-2483.

33. Sharma MD, Hou DY, Liu Y, Koni PA, Metz R, et al. (2009) Indoleamine 2,3-dioxygenase controls conversion of Foxp3+ Tregs to TH17-like cells in tumor-draining lymph nodes. Blood 113: 6102-6111.

34. Yan Y, Zhang GX, Gran B, Fallarino F, Yu S, et al. (2010) IDO upregulates regulatory $T$ cells via tryptophan catabolite and suppresses encephalitogenic $\mathrm{T}$ cell responses in experimental autoimmune encephalomyelitis. J Immuno 185: 5953-5961.

35. Terness P, Bauer TM, Röse L, Dufter C, Watzlik A, et al. (2002) Inhibition of allogeneic $T$ cell proliferation by indoleamine 2,3-dioxygenase-expressing dendritic cells: mediation of suppression by tryptophan metabolites. J Exp Med 196: 447-457.

36. Zhang X, Jin J, Peng X, Ramgolam VS, Markovic-Plese S (2008) Simvastatin inhibits IL-17 secretion by targeting multiple IL-17-regulatory cytokines and by inhibiting the expression of IL-17 transcription factor RORC in CD4+ lymphocytes. J Immunol 180: 6988-6996. 
Citation: Yang K, Chi H (2014) Metabolic Control of Th17 Cell Generation and CNS Inflammation. J Neurol Neurophysiol S12: 004. doi:10.4172/21559562.S12-004

37. Zhang X, Tao Y, Wang J, Garcia-Mata R, Markovic-Plese S. (2013) Simvastatin Inhibits Secretion of Th17-Polarizing Cytokines and Antigen Presentation by Dcs in Patients with Relapsing Remitting Multiple Sclerosis. Eur J Immuno 43: $281-289$

38. Zhang X, Tao Y, Troiani L, Markovic-Plese S (2011) Simvastatin inhibits IFN regulatory factor 4 expression and Th17 cell differentiation in CD4+ T cells derived from patients with multiple sclerosis. J Immunol 187: 3431-3437.

39. Youssef S, Stüve O, Patarroyo JC, Ruiz PJ, Radosevich JL, et al. (2002) The HMG-CoA reductase inhibitor, atorvastatin, promotes a Th2 bias and reverses paralysis in central nervous system autoimmune disease. Nature 420: 78-84.

40. Weber MS, Youssef S, Dunn SE, Prod'homme T, Neuhaus O, et al. (2006) Statins in the treatment of central nervous system autoimmune disease. J Neuroimmunol 178: 140-148.

41. Stüve O, Youssef S, Weber MS, Nessler S, von Büdingen HC, et al. (2006) Immunomodulatory synergy by combination of atorvastatin and glatiramer acetate in treatment of CNS autoimmunity. J Clin Invest 116: 1037-1044.

42. Zhao C, Dahlman-Wright K (2010) Liver X receptor in cholesterol metabolism. J Endocrinol 204: 233-240.

43. Xu J, Wagoner G, Douglas JC, Drew PD (2009) Liver X receptor agonist regulation of Th17 lymphocyte function in autoimmunity. J Leukoc Biol 86: 401409 .

44. Cui G, Qin X, Wu L, Zhang Y, Sheng X, et al. (2011) Liver X receptor (LXR) mediates negative regulation of mouse and human Th17 differentiation. $\mathrm{J}$ Clin Invest 121: 658-670.

45. Klotz L, Burgdorf S, Dani I, Saijo K, Flossdorf J, et al. (2009) The nuclear receptor PPAR gamma selectively inhibits Th17 differentiation in a $T$ cellintrinsic fashion and suppresses CNS autoimmunity. J Exp Med 206: 20792089.

46. Dunn SE, Bhat R, Straus DS, Sobel RA, Axtell R, et al. (2010) Peroxisome Proliferator-Activated Receptor Delta Limits the Expansion of Pathogenic Th Cells During Central Nervous System Autoimmunity. J Exp Med 207: 15991608.

47. Kanakasabai S, Walline CC, Chakraborty S, Bright JJ (2011) PPARס deficient mice develop elevated Th1/Th17 responses and prolonged experimental autoimmune encephalomyelitis. Brain Res 1376: 101-112.

48. Kleinewietfeld M, Manzel A, Titze J, Kvakan H, Yosef N, et al. (2013) Sodium chloride drives autoimmune disease by the induction of pathogenic $\mathrm{TH} 17$ cells. Nature 496: 518-522.

49. Wu C, Yosef N, Thalhamer T, Zhu C, Xiao S, et al. (2013) Induction of pathogenic TH17 cells by inducible salt-sensing kinase SGK1. Nature 496: 513-517.

50. Huang G, Wang Y, Vogel P, Kanneganti TD, Otsu K, et al. (2012) Signaling via the kinase p38a programs dendritic cells to drive $\mathrm{TH} 17$ differentiation and autoimmune inflammation. Nat Immunol 13: 152-161.
This article was originally published in a special issue, Neurodegenerative Diseases: Sympłoms and Therapeutics handled by Editor(s). Dr. Jin J Luo, Temple University School of Medicine, USA 